\title{
La fièvre chez le voyageur de retour au pays Lignes directrices pour l'évaluation initiale
}

\section{Préambule}

Le Comité consultatif de la médecine tropicale et de la médecine des voyages (CCMTMV) fournit à l'Agence de la santé publique du Canada (ASPC) des conseils constants et à jour de nature médicale, scientifique et de santé publique concernant les maladies tropicales infectieuses et les risques pour la santé associés aux voyages internationaux. L'ASPC reconnaît que les conseils et les recommandations figurant dans le présent document reposent sur la pratique médicale et les connaissances scientifiques les plus récentes, et les diffuse dans le but d'informer les voyageurs ainsi que les professionnels de la santé qui sont appelés à leur fournir des soins.

Les personnes qui administrent ou utilisent des médicaments, des vaccins ou d'autres produits devraient également bien connaître la monographie du produit ou toute autre norme ou instruction approuvée concernant leur usage. Les recommandations relatives à l'usage des produits et les autres renseignements présentés ici peuvent différer de ceux qui figurent dans la monographie ou toute autre norme ou instruction approuvée établie par les fabricants autorisés. Les fabricants ont fait approuver leurs produits et en ont démontré l'innocuité et l'efficacité uniquement lorsqu'ils sont utilisés conformément à la monographie ou à toute autre norme ou instruction approuvée semblable.

\section{Introduction}

La fièvre chez le voyageur international est un syndrome couramment observé lors d'un retour de voyage et elle peut ouvrir la voie à une maladie grave et potentiellement mortelle, la plus importante étant le paludisme. Par conséquent, il convient de demander à tous les patients fébriles ou qui se plaignent de fièvre s'ils ont voyagé récemment. Même si ce type de fièvre peut être attribuable à des infections bénignes spontanément résolutives, comme celles causées par les agents courants de la diarrhée du voyageur, ou à des causes typiques largement répandues dans le monde et non liées au voyage, il faut d'abord l'interpréter comme une urgence médicale qui justifie une évaluation immédiate et exhaustive. Pour poser un diagnostic précis et assurer une prise en charge adéquate, il faut connaître les antécédents complets de voyage, notamment les détails concernant l'itinéraire (destination, dates d'arrivée et de départ), le mode de tourisme pratiqué, les préparatifs

\footnotetext{
*Membres : Dr P. J. Plourde (président); Dr C. Beallor; Dr A. Boggild; Dr J. Brophy; Dr M. Crockett; Dr W. Ghesquiere; Ms A. Henteleff; Dr A. McCarthy; Dr K. L. McClean Représentants d'office : Dr G. Brunette; Dr P. Charlebois; Dr J. Creaghan, Dr J. Given; Dr P. McDonald; Dr M. Tepper Représentants de liaison : Dr C. Greenaway; Dr C. Hui; Dr A. Pozgay; Dr P. Teitelbaum

Membre émerite : Dr C. William L. Jeanes

Consultant : Dr S. Schofield
}

$†$ La présente déclaration a été préparée par $D^{r} A$. Boggild, Dr W. Ghesquiere, et $D^{r}$ A. McCarthy et approuvée par le CCMTMV. 
avant le départ, de même que les risques d'exposition ou les traitements ou soins médicaux reçus à l'étranger et un examen physique complet, en plus d'accorder une attention particulière aux appareils ou systèmes présentant des signes ou des symptômes de localisation. Cette évaluation devrait être suivie de l'utilisation judicieuse d'analyses de laboratoire réalisées au moyen des tests minimaux essentiels énoncés dans l'algorithme par étapes présenté plus loin.
Le présent document comporte deux objectifs :

- Orienter les cliniciens de première ligne, notamment ceux qui travaillent dans les services d'urgence, dans les cliniques sans rendez-vous et dans les cabinets de soins primaires, dans l'évaluation et la prise en charge initiales de la fièvre chez le voyageur de retour au pays.

- Fournir aux cliniciens de première ligne qui n'ont pas facilement accès à des services d'hospitalisation, de médecine interne ou d'infectiologie des directives sur l'évaluation et la prise en charge de la fièvre chez le voyageur de retour au pays au-delà de l'étape initiale.

\section{Épidémiologie de la fièvre chez le voyageur de retour au pays}

La fièvre chez le voyageur de retour au pays peut être attribuable à des maladies ou à des infections tropicales qui sont largement répandues dans le monde. Dans bien des grandes séries de maladies survenues après un voyage à l'étranger, le paludisme (20 à $30 \%)^{1}$, la diarrhée aiguë du voyageur (10 à $20 \%$ ) et les infections des voies respiratoires (10 à $15 \%$ ) ont souvent été considérées comme les causes principales de la fièvre chez les voyageurs ${ }^{2-11}$ (tableau 1). Parmi les autres causes fréquentes de la fièvre chez le voyageur de retour au pays, mentionnons la dengue (5\%), la fièvre entérique provoquée par le sérovar Typhi ou Paratyphi de Salmonella enterica (2 à $7 \%$ ), les infections de la peau et des tissus mous (2 à $11 \%$ ), les rickettsioses (3\%), les infections des voies urinaires et les infections transmissibles sexuellement (2 à $3 \%$ ), les hépatites virales (3\%) et le syndrome viral non spécifique ou le syndrome apparenté à la mononucléose (4 à $25 \%)^{2-11}$. Chez les jeunes voyageurs de retour au pays qui présentent de la fièvre, le paludisme (35\%), les syndromes viraux (28\%), les maladies fébriles non spécifiques (11\%), la dengue (6\%) et la fièvre entérique (6\%) sont les étiologies les mieux représentées ${ }^{12}$. Une fièvre survenant après un voyage à l'étranger peut également être due à des causes non infectieuses et englober diverses étiologies telles que des réactions aux médicaments, une embolie pulmonaire, des affections inflammatoires comme la maladie intestinale inflammatoire, une tumeur maligne et l'hyperthyroïdie.
Une analyse axée sur la destination permet de déterminer l'épidémiologie de la maladie contractée en voyage ${ }^{13}$ (tableau 2). Des antécédents de voyage dans le sous-continent indien chez une personne fébrile de retour au pays devraient éveiller des soupçons de fièvre entérique : sur les 416 cas de typhoïde importés au Royaume-Uni au cours d'une période de trois ans, $70 \%$ provenaient de l'Inde et du Pakistan ${ }^{14}$. Le paludisme, par contre, se caractérise par une épidémiologie très différente chez les cas importés, les risques relatifs chez les voyageurs étant les plus élevés en Afrique subsaharienne ${ }^{15}$. Contrairement au paludisme, la dengue se contracte le plus souvent en Asie du Sud, en Asie du Sud-Est et en Amérique latine plutôt qu'en Afrique ${ }^{13}$.

Le but du voyage représente un autre renseignement utile des antécédents de voyage. Les personnes qui voyagent pour rendre visite à des amis ou à des parents, y compris les enfants, constituent un groupe particulièrement à risque de contracter des infections liées au voyage ${ }^{12}$, en particulier le paludisme ${ }^{16}$ et la fièvre entérique ${ }^{4}$ et, dans le cas des enfants, l'hépatite virale aiguë ${ }^{17}$.

Un portrait exhaustif des antécédents de voyage ainsi qu'une bonne compréhension des maladies potentiellement présentes selon les dates et le mode de tourisme pratiqué, les risques géographiques et l'itinéraire de voyage précis, notamment les activités et les comportements, sont essentiels 
pour restreindre le diagnostic différentiel et écarter les infections potentiellement mortelles contractées en voyage. La période d'incubation, c'est-à-dire le temps écoulé entre l'exposition et l'infection initiales et l'apparition des symptômes, permet également de préciser le diagnostic différentiel. Par exemple, les maladies contractées en voyage à courte période d'incubation, notamment la dengue, le chikungunya et la diarrhée du voyageur, peuvent être écartées de façon fiable si aucun symptôme ne s'est manifesté plus de deux semaines après le départ de la région d'endémicité. De même, les maladies contractées en voyage à longue période d'incubation comme la tuberculose, l'hépatite B ou la leishmaniose viscérale peuvent être exclues (comme étant liées au voyage) si les symptômes apparaissent quelques jours après le retour d'un voyage d'une semaine à l'étranger. Il est important de tenir compte des dates d'exposition possibles (au plus tôt et au plus tard) chez les personnes revenant d'un court ou d'un long voyage afin de mieux guider l'utilisation de la période d'incubation lors de l'établissement d'un diagnostic différentiel. Lalgorithme par étapes qui suit est conçu de manière à orienter le clinicien dans son évaluation initiale d'un voyageur de retour au pays qui présente de la fièvre et, exceptionnellement, à fournir des directives supplémentaires lorsque des services d'hospitalisation ou la consultation d'un spécialiste sont retardées ou non accessibles.

Tableau 1 : Principaux diagnostics spécifiques par des analyses sur la fièvre chez le voyageur de retour au pays réalisées dans un seul centre ${ }^{2 ; 3 ; 5-11}$ et dans plusieurs centres ${ }^{4 ; 12}$

\begin{tabular}{|c|c|}
\hline 1. Paludisme (20 à $30 \%)$ & 6. Infections de la peau et des tissus mous (2 à $11 \%$ ) \\
\hline $\begin{array}{l}\text { 2. Diarrhée aiguë du voyageur / gastroentérite } \\
(10 \text { à } 20 \%)\end{array}$ & $\begin{array}{l}\text { 7. Rickettsioses, y compris la fièvre à tique africaine, la fièvre } \\
\text { boutonneuse méditerranéenne (typhus à tique) (3\%) }\end{array}$ \\
\hline 3. Infections des voies respiratoires (IVR) (10 à $15 \%$ ) & $\begin{array}{l}\text { 8. Infection aiguë des voies urinaires / infections transmissibles } \\
\text { sexuellement (ITS) (2 à } 3 \% \text { ) }\end{array}$ \\
\hline 4. Dengue ( $5 \%)$ & 9. Hépatite virale (3\%) \\
\hline $\begin{array}{l}\text { 5. Fièvre entérique (sérovar Typhi ou Paratyphi } \\
\text { de S. enterica) (2 à } 7 \% \text { ) }\end{array}$ & $\begin{array}{l}\text { 10. Syndrome d'allure virale ou apparenté à la mononucléose } \\
\text { (4 à } 25 \% \text { ) }\end{array}$ \\
\hline
\end{tabular}

(Il est possible de consulter les déclarations du CCMTMV sur le site http://www.phac-aspc.gc.ca/tmp-pmv/catmat-ccmtmv/ index-fra.php)

Tableau 2 : Principaux diagnostics spécifiques par des analyses multicentriques ${ }^{4 ; 13}$ sur la fièvre chez le voyageur de retour au pays, par région de voyage

\begin{tabular}{|c|c|}
\hline Afrique subsaharienne & $\begin{array}{l}\text { Paludisme, IVR, maladie diarrhéique, } \\
\text { infections de la peau et des tissus mous }\end{array}$ \\
\hline Asie du Sud-Est & Dengue, IVR, maladie diarrhéique, paludisme \\
\hline Asie centrale du Sud / sous-continent indien & Fièvre entérique, dengue, paludisme, maladie diarrhéique \\
\hline Amérique du Sud & Maladie diarrhéique, IVR, dengue, paludisme \\
\hline Caraïbes / Amérique centrale & Maladie diarrhéique, IVR, dengue, paludisme \\
\hline
\end{tabular}




\section{Évaluation de la fièvre chez le voyageur de retour au pays}

Objectif : Écarter les maladies potentiellement mortelles, hautement transmissibles ou traitables.

ÉTAPE 1 : Antécédents de voyage

A. PRÉPARATIFS AVANT LE DÉPART
Consultation avant le départ : date, lieu, coordonnées

Vaccins reçus et dates : forme orale ou injectable, s'il y a lieu; série complète de vaccins de voyage et ceux offerts systématiquement aux enfants

Prophylaxie du paludisme : médicament, dose, calendrier, respect du traitement, durée, effets secondaires

Autre prophylaxie : médicament, dose, calendrier, respect du traitement, durée, effets secondaires

Autres mesures de protection individuelle : traitement de réserve du paludisme /de la diarrhée du voyageur (DV); moustiquaires de lit; vêtements; emploi d'insecticide

Préparatifs en vue du transport aérien (prophylaxie de la thrombose veineuse profonde, médicaments contre le décalage horaire)

Préparatifs contre les risques environnementaux (soleil, chaleur extrême, altitude)

\section{B. ITINÉRAIRE DE VOYAGE PARTICULIER}

Dates de voyage (approximation de la période d'incubation)

Saison (pluvieuse, sèche)

Destinations particulières (régions; milieu urbain, milieu rural; eau douce à proximité; jungle, désert)

Motif du voyage (tourisme, affaires, visite d'amis ou de parents, autre)

Mode de tourisme (hébergement; hors des sentiers touristiques; camping, trekking)

Population locale (possibilité de tuberculose [TB] : maladie, éclosions, contacts avec des cas)

Transport (véhicules bondés; utilisation d'animaux comme le chameau ou l'éléphant) 


\section{ANTÉCÉDENTS D'EXPOSITION*}

Aliments de rue / eau locale (fièvre entérique, DV)

Morsures d'arthropodes (paludisme, dengue, chikungunya, arboviroses, rickettsioses, trypanosomiase africaine)

Viande crue / produits laitiers non pasteurisés (trichinose, brucellose, toxoplasmose)

Exposition à du sang et à des liquides organiques lors de rapports sexuels, tatouages, perçages, injections, y compris les vaccins, les injections par voie intraveineuse et la prophylaxie post-exposition contre la rage (VIH, VHB, VHC, VHS, syphilis, GC/CT)

Activités en eau douce comme la baignade, le kayak, la descente en eau vive (schistosomiase, leptospirose)

Exposition aux animaux (fièvre Q, brucellose, tularémie, charbon, rage, fièvre hémorragique de Crimée-Congo)

Safari (rickettsiose, trypanosomiase africaine)

* Pour connaître l'épidémiologie de certaines maladies en particulier, veuillez consulter les sites Web de l'Organisation mondiale de la Santé (OMS) (http://www.who.int/topics/fr/), de l'Agence de la santé publique du Canada (ASPC) (http://www.phac-aspc.gc.ca/tmp-pmv/info/index-fra.php) ou des Centers for Disease Control and Prevention (CDC) des États-Unis (http://wwwnc.cdc.gov/travel/content/diseases.aspx).

\section{ÉTAPE 2 : Antécédents de fièvre et tableau clinique}

\begin{tabular}{|c|c|c|}
\hline \multirow[t]{9}{*}{ A. FORME DE FIÈVRE } & \multirow{3}{*}{$\begin{array}{l}\text { Fièvre quotidienne/ } \\
\text { continue }\end{array}$} & Fréquent : paludisme, DV, IVR, fièvre entérique \\
\hline & & Peu fréquent : rickettsioses \\
\hline & & Rare : VIH, fièvre Q, brucellose, TB \\
\hline & \multirow[t]{3}{*}{ Fièvre biphasique } & Fréquent : paludisme, dengue \\
\hline & & Peu fréquent : leptospirose \\
\hline & & Rare : fièvre jaune, arbovirus \\
\hline & \multirow[t]{3}{*}{ Fièvre récurrente } & Fréquent : paludisme, fièvre entérique \\
\hline & & Peu fréquent : abcès hépatique amibien, leptospirose \\
\hline & & $\begin{array}{l}\text { Rare : VIH, fièvre Q, brucellose, TB, mycoses endémiques, } \\
\text { borréliose }\end{array}$ \\
\hline
\end{tabular}




\begin{tabular}{|c|c|c|}
\hline \multirow{9}{*}{$\begin{array}{l}\text { B. PÉRIODE D'INCUBATION } \\
\text { (DÉFINITION : TEMPS ÉCOULÉ } \\
\text { ENTRE L'EXPOSITION / } \\
\text { INFECTION ET L'APPARITION } \\
\text { DE LA MALADIE) }\end{array}$} & \multirow[t]{3}{*}{$<2$ semaines } & Fréquent : paludisme, DV, dengue, IVR, grippe \\
\hline & & Peu fréquent : rickettsioses, méningite \\
\hline & & Rare : fièvre jaune, arbovirus, fièvre hémorragique virale \\
\hline & \multirow[t]{3}{*}{2 à 6 semaines } & Fréquent : paludisme, fièvre entérique \\
\hline & & $\begin{array}{l}\text { Peu fréquent : virus de l'hépatite } \mathrm{A}(\mathrm{VHA}) \text {, fièvre de Katayama, } \\
\text { abcès hépatique amibien, virus de l'hépatite } \mathrm{E} \text { (VHE) }\end{array}$ \\
\hline & & Rare : VIH, fièvre Q, brucellose, trypanosomiase d'Afrique de l'Est \\
\hline & \multirow[t]{3}{*}{$>6$ semaines } & Fréquent : paludisme \\
\hline & & Peu fréquent : VHB, abcès hépatique amibien, TB \\
\hline & & $\begin{array}{l}\text { Rare : VIH, leishmaniose viscérale, mycoses endémiques, } \\
\text { trypanosomiase d'Afrique de l'Ouest, VHC }\end{array}$ \\
\hline \multirow{9}{*}{$\begin{array}{l}\text { C. DURÉE DE LA FIÈVRE } \\
\text { AU MOMENT DE LA } \\
\text { CONSULTATION }\end{array}$} & \multirow[t]{3}{*}{$<7$ jours } & Fréquent : paludisme, DV, dengue, fièvre entérique, IVR \\
\hline & & Peu fréquent : rickettsioses, leptospirose, méningite \\
\hline & & Rare : fièvre jaune, arbovirus \\
\hline & \multirow[t]{3}{*}{7 à 21 jours } & Fréquent : paludisme, fièvre entérique \\
\hline & & Peu fréquent : rickettsioses, hépatite virale, leptospirose \\
\hline & & Rare : VIH, fièvre Q, brucellose \\
\hline & \multirow[t]{3}{*}{$>21$ jours } & Fréquent : paludisme, fièvre entérique \\
\hline & & Peu fréquent : TB, VHB, endocardite bactérienne \\
\hline & & Rare : VIH, fièvre Q, brucellose \\
\hline
\end{tabular}

Arbovirus : Les arbovirus comprennent d'autres virus transmis par les moustiques comme le virus du chikungunya, le virus Mayaro, le virus O'nyong-nyong, le virus de la rivière Ross, le virus Sindbis, le virus de l'encéphalite équine, le virus du Nil occidental, le virus de La Crosse, le virus Oropouche, le virus de la fièvre de la vallée du Rift et des virus transmis par les tiques comme le virus de la forêt Kyasanur, le virus Omsk et le virus de la fièvre hémorragique de Crimée-Congo. 


\section{ÉTAPE 3 : Premières analyses de laboratoire après un examen physique complet, y compris un examen exhaustif de la peau}

\section{Toute personne qui présente de la fièvre au retour d'un voyage devrait se soumettre au moins aux analyses suivantes :}

Chez tout voyageur fébrile en provenance d'une région où le paludisme est endémique ${ }^{1}$, il faut considérer le diagnostic de paludisme jusqu'à preuve du contraire. Les antécédents de voyages devraient être mentionnés sur toutes les demandes d'analyses de laboratoire.

1. Formule sanguine et tests différentiels; enzymes hépatiques; électrolytes; créatinine.

2. Frottis sanguin (paludisme) avec ou sans bandelette réactive de détection des antigènes, au moins 3 fois sur une période de 24 à 48 heures.

3. Hémocultures x 2 (le sérovar Typhi ou Paratyphi de S. enterica; méningocoques; agents courants de bactériémie.

4. Analyse des urines (avant ou sans culture d'urine).

\section{Envisager les tests supplémentaires suivants selon les antécédents et l'épidémiologie :}

5. Coproculture pour la recherche d'entéropathogènes x 1 (Salmonella, Shigella, Campylobacter, Yersinia, E. coli O157:H7).

6. Radiographie pulmonaire.

7. Recherche d'œufs et de parasites dans les selles (Cyclospora, Cryptosporidium, Entamoeba histolytica, Giardia).

8. Épreuve sérologique de la dengue si la période d'incubation probable est inférieure à 2 semaines ET que la personne revient d'Asie du Sud, d'Asie du Sud-Est ou d'Amérique latine.

9. Le tube contenant le sérum prélevé en phase aiguë doit être conservé au laboratoire et apparié avec des échantillons de sérum en phase de convalescence s'il n'y a pas de diagnostic dans les 10 à 14 jours.

**Il faut envisager d'adresser le patient à un spécialiste de la médecine tropicale ou des maladies infectieuses (selon la disponibilité) si les premières analyses de laboratoire ne permettent pas de poser un diagnostic définitif et que l'état de santé du patient ne s'améliore pas ou que ce dernier est à risque de présenter des complications (femmes enceintes, enfants, affections concomitantes sous-jacentes, personnes immunodéprimées, etc.). Au besoin, il est possible d'obtenir de l'aide supplémentaire par l'intermédiaire du Réseau canadien sur le paludisme (http://www.phac-aspc.gc.ca/tmp-pmv/quinine/ index-fra.php)** 
ÉTAPE 4 : Prise en charge empirique des cas soupçonnés de diarrhée du voyageur ou de fièvre entérique avec ou sans rickettsiose chez des voyageurs adultes $\mathrm{i}$, ii

Déterminer si le patient satisfait à tous les critères suivants

1. Fièvre non différenciée;

2. Résultat négatif des frottis sanguins (paludisme) ${ }^{1}$;

3. Létat de santé ne s'est pas amélioré depuis les 48 à 72 dernières heures;

4. Tous les autres tests et cultures ont été demandés et sont en attente ou non contributifs.

\begin{tabular}{|c|c|}
\hline Voyage dans le sous-continent indien ou en Asie du Sud-Est & $\begin{array}{l}\text { Voyage à l'extérieur du sous-continent indien ou de l'Asie du } \\
\text { Sud-Est }\end{array}$ \\
\hline $\begin{array}{l}\text { 1. Azithromycine } 1000 \mathrm{mg} \text { par voie orale une fois par jour } \\
\text { x } 5 \text { jours (ou } 1000 \mathrm{mg} \text { une fois le premier jour, puis } \\
500 \mathrm{mg} \text { une fois par jour } \mathrm{x} 6 \text { jours) avec ou sans } \\
\text { 2. Doxycycline** } 100 \mathrm{mg} \text { par voie orale deux fois par jour } \\
\text { x } 7 \text { jours. }\end{array}$ & $\begin{array}{l}\text { 1. Ciprofloxacine } 500 \mathrm{mg} \text { par voie orale deux fois par jour } \\
\text { x } 3 \text { à } 7^{*} \text { jours avec ou sans } \\
\text { 2. Doxycycline } \text { D }^{* *} 100 \mathrm{mg} \text { par voie orale deux fois par jour } \\
\text { x } 7 \text { jours. }\end{array}$ \\
\hline
\end{tabular}

i) Létape 4 ne remplace pas l'étape 3 (Premières analyses de laboratoire après un examen physique complet), et le traitement empirique ne devrait pas empêcher la réalisation d'un suivi étroit et constant du patient. Par fièvre non différenciée, on entend une fièvre sans symptômes ou signes de localisation dominants. C'est souvent le motif de consultation dans bien des cas de maladies contractées en voyage, dont le paludisme et la fièvre entérique. Il est capital que tout voyageur fébrile de retour d'une région potentiellement impaludée sois soumis à un test de détection du paludisme composé de 2 ou 3 frottis sanguins sur une période de 48 heures, avec ou sans test rapide de détection des antigènes. Les patients adultes dont l'état ne s'est pas grandement amélioré, selon une évaluation subjective ou objective, au cours des 48 à 72 heures précédentes peuvent recevoir le traitement empirique susmentionné en attendant les résultats des analyses. Ces patients ont besoin d'une réévaluation clinique fréquente (tous les jours ou aux deux jours) jusqu'à ce qu'un diagnostic soit posé et qu'un traitement définitif soit instauré, ou jusqu'à ce qu'on observe une amélioration clinique subjective et objective. Avant d'amorcer le traitement empirique susmentionné, il faut s’assurer d'avoir obtenu tous les échantillons destinés aux tests microbiologiques comme il est indiqué à l'étape 3.

ii) Il convient de faire preuve de prudence lors de l'évaluation et du traitement empirique de la fièvre chez les enfants, compte tenu du taux plus élevé de complications dans ce groupe d'âge. Les cliniciens devraient établir un faible seuil pour l'admission et l'amorce du traitement par voie intraveineuse (IV) contre la fièvre entérique soupçonnée, auquel cas l'administration parentérale de céphalosporines de troisième génération (ceftriaxone ou céfotaxime) pourrait être envisagée. De même, l'utilisation de doxycycline et de ciprofloxacine n'est pas habituelle chez les enfants et on la réserve généralement à des cas particuliers pour lesquels les avantages de ces antibiotiques semblent dépasser les risques potentiels (coloration permanente des dents, arthropathie). Il faut envisager d'adresser le patient à un spécialiste dans le traitement des maladies tropicales chez les enfants.

\footnotetext{
*Schéma thérapeutique de 3 jours pour les cas soupçonnés de diarrhée du voyageur; schéma thérapeutique de 7 à 10 jours pour les cas soupçonnés de fièvre entérique.

**Cela s'applique également à la leptospirose.
} 


\section{Annexe I. Tests spécifiques visant à exclure la présence d'une infection fréquente contractée en voyage pouvant causer de la fièvre}

\begin{tabular}{|c|c|}
\hline Infection contractée en voyage & Test diagnostique \\
\hline Paludisme & $\begin{array}{l}\text { 1. Frottis sanguins (étalement mince et goutte épaisse) avec ou sans épreuve par bandelette } \\
\text { réactive de détection des antigènes; minimum } 2 \text { ou } 3 \text { fois sur une période de } 24 \text { à } 48 \text { heures. } \\
\text { 2. Échantillon de sang pour la détection du paludisme par l'amplification par la polymérase } \\
\text { (PCR) dans le cas d'un résultat négatif des frottis et/ou de la bandelette réactive, mais d'un } \\
\text { indice de suspicion élevé. }\end{array}$ \\
\hline $\begin{array}{l}\text { Diarrhée aiguë du voyageur } \\
\text { / gastroentérite ( } 60 \text { à } 80 \text { \% } \\
\text { d'origine bactérienne) }\end{array}$ & $\begin{array}{l}\text { 1. Coproculture pour la recherche d'entéropathogènes x } 1 \text { (détection de Salmonella, Shigella, } \\
\text { Campylobacter, E. coli O157:H7 et souvent Yersinia). } \\
\text { 2. Recherche de la toxine de Clostridium difficile dans les selles. } \\
\text { 3. Recherche d'œufs et de parasites dans les selles x } 3 \text { (il faut savoir que ce ne sont pas tous les } \\
\text { laboratoires qui effectuent systématiquement des tests de détection des protozoaires, notam- } \\
\text { ment les coccidies; il convient de vérifier auprès du laboratoire local les exigences applicables } \\
\text { aux demandes de coloration spéciales). } \\
\text { 4. Sérologie amibienne avec ou sans test ELISA pour la recherche d'Entamoeba histolytica dans } \\
\text { les selles, en cas de présence de sang dans les selles. }\end{array}$ \\
\hline $\begin{array}{l}\text { Infection des voies } \\
\text { respiratoires }\end{array}$ & $\begin{array}{l}\text { 1. Radiographie pulmonaire. } \\
\text { 2. Écouvillonnage du nasopharynx en vue de la réalisation d'un test de détection des antigènes } \\
\text { ou d'un test par PCR (virus grippal A/B, virus respiratoire syncytial, adénovirus, virus para- } \\
\text { influenza de type } 1 \text { à } 3 \text {, métapneumovirus humain, coronavirus). } \\
\text { 3. Échantillons d'expectorations pour cultures et épreuves de sensibilité, et recherche de bacilles } \\
\text { acidorésistants (conformément à l'indice de suspicion). } \\
\text { 4. Détection de l'antigène de Legionella dans les urines. } \\
\text { 5. Virus d'Epstein-Barr (VEB) : le test Monospot est peu fiable chez les enfants } \leq 4 \text { ans; IgM/IgG } \\
\text { anti-VCA du VEB, IgM/IgG anti-ANEB. } \\
\text { 6. (Épreuve sérologique pour la fièvre Q, Histoplasma, Blastomyces, Coccidioides conformé- } \\
\text { ment à l'indice de suspicion et aux expositions associées au voyage); détection de l'antigène } \\
\text { d'Histoplasma dans les urines). }\end{array}$ \\
\hline Dengue & $\begin{array}{l}\text { 1. Sérum en phase aiguë et en phase de convalescence ( } 2 \text { semaines plus tard) pour la recherche } \\
\text { d'IgM et d'IgG dirigés contre le virus de la dengue. }\end{array}$ \\
\hline $\begin{array}{l}\text { Fièvre entérique due au } \\
\text { sérovar Typhi ou Paratyphi } \\
\text { de Salmonella enterica }\end{array}$ & $\begin{array}{l}\text { 1. Hémoculture x } 2 \text { (faire attention si le patient a reçu des antibiotiques, ce qui peut entraîner } \\
\text { un résultat négatif). } \\
\text { 2. Coproculture. } \\
\text { 3. (Prélèvement de moelle osseuse par aspiration et culture). }\end{array}$ \\
\hline
\end{tabular}




\begin{tabular}{|c|c|}
\hline $\begin{array}{l}\text { Infection de la peau et des } \\
\text { tissus mous }\end{array}$ & $\begin{array}{l}\text { 1. Diagnostic clinique. } \\
\text { 2. Écouvillonnage cutané pour la recherche de SASM et de SARM si la lésion est exsudative. } \\
\text { 3. Dans le cas d'une lésion ulcérative, frottis colorés au Giemsa, biopsie ou aspiration pour } \\
\text { culture de Leishmania ou PCR; envisager un écouvillonnage cutané pour exclure un ecthyma } \\
\text { dû à Staphylococcus ou à Pseudomonas. }\end{array}$ \\
\hline Rickettsioses & $\begin{array}{l}\text { 1. Diagnostic clinique - la présence d'une escarre constitue un diagnostic (mais peut ne pas } \\
\text { être présente). } \\
\text { 2. Sérum en phase aiguë et en phase de convalescence pour la détection de rickettsies. }\end{array}$ \\
\hline $\begin{array}{l}\text { Infection aiguë des voies } \\
\text { urinaires / infection } \\
\text { transmissible sexuellement }\end{array}$ & $\begin{array}{l}\text { 1. Analyse et examen microscopique des urines. } \\
\text { 2. Culture d'urine. } \\
\text { 3. Prélèvement d'urine et/ou écouvillonnage endocervical pour la détection de la gonorrhée et } \\
\text { de l'infection à Chlamydia. } \\
\text { 4. Écouvillonnage des vésicules génitales pour la détection d'agents viraux par PCR. } \\
\text { 5. Échantillons de sang pour épreuves sérologiques de détection du VIH, du VHB, du VHC et } \\
\text { de la syphilis. }\end{array}$ \\
\hline Hépatite virale & $\begin{array}{l}\text { 1. Virus de l'hépatite A : IgM anti-VHA, IgG anti-VHA (sauf s'il y a des antécédents de vaccination). } \\
\text { 2. Virus de l'hépatite B : HBsAg (antigène de surface), HBsAb (anticorps de surface), HBcAb } \\
\text { (anticorps nucléocapsidique), HBeAg (antigène e), HBeAb (anticorps e); ADN du VHB. } \\
\text { 3. Virus de l'hépatite C : anticorps totaux anti-VHC. } \\
\text { 4. Virus de l'hépatite D : anticorps anti-VHD; sérum pour la détection de l'ARN du VHD par } \\
\text { RT-PCR. } \\
\text { 5. Virus de l'hépatite E : anticorps IgM anti-VHE; échantillons de sang ou de selles pour la } \\
\text { détection du VHE par PCR. } \\
\text { 6. Virus d'Epstein-Barr virus (VEB) : le test Monospot est peu fiable chez les enfants } \leq 4 \text { ans; } \\
\text { IgM/IgG anti-VCA du VEB, IgM/IgG anti-ANEB. } \\
\text { 7. Cytomégalovirus (CMV) : IgM/IgG anti-CMV; antigénémie du CMV; sérum pour la détection } \\
\text { du CMV par PCR. }\end{array}$ \\
\hline $\begin{array}{l}\text { Autres infections } \\
\text { potentiellement contractées } \\
\text { en voyage diagnostiquées par } \\
\text { analyses sérologiques }\end{array}$ & $\begin{array}{l}\text { 1. Infections virales : virus du chikungunya, arbovirus. } \\
\text { 2. Infections bactériennes : fièvre } \mathrm{Q} \text {, infection à Brucella (la culture peut également être faite à } \\
\text { partir de sang ou de moelle osseuse), infection à Leptospira. } \\
\text { 3. Infections fongiques : infections à Histoplasma, Blastomyces, Coccidioides, Cryptococcus } \\
\text { (détection possible par analyse du sérum ou du liquide céphalorachidien, ou détection de } \\
\text { l'antigène dans les urines). } \\
\text { 4. Infections parasitaires : infections à Strongyloides ou à Schistosoma, amibiose (détection } \\
\text { possible par la recherche d'œufs et de parasites dans les selles et par test ELISA à partir } \\
\text { d'échantillons de selles). }\end{array}$ \\
\hline
\end{tabular}




\section{Annexe II. Ressources supplémentaires d'orientation sur la fièvre chez le voyageur de retour au pays}

\section{Déclarations du Comité consultatif de la médecine tropicale et de la médecine des voyages (CCMTMV)}

Recommandations canadiennes pour la prévention et le traitement du Paludisme (Malaria) chez les voyageurs internationaux (2009) :

http://www.phac-aspc.gc.ca/publicat/ccdr-rmtc/09vol35/35sl/index-fra.php

Déclaration sur la diarrhée persistante après un voyage (2006) :

http://www.phac-aspc.gc.ca/publicat/ccdr-rmtc/06vol32/acs-01/index-fra.php

Déclaration sur les risques d'infections transmises sexuellement chez les voyageurs (2006):

http://www.phac-aspc.gc.ca/publicat/ccdr-rmtc/06vol32/acs-05/index-fra.php

\section{Agence de la santé publique du Canada}

Conseils de santé aux voyageurs :

http://www.phac-aspc.gc.ca/tmp-pmv/pub-fra.php

Laboratoire nationale de microbiologie :

http://www.nml-lnm.gc.ca/index-fra.htm

Coordonateurs du Réseau canadien sur le paludisme, accessible sur le site Web de l'Agence de la santé publique du Canada :

http://www.phac-aspc.gc.ca/tmp-pmv/quinine/index-fra.php

\section{Centers for Disease Control and Prevention des États-Unis (en anglais) :}

http://www.cdc.gov/

Viral Special Pathogens Branch, NCID (en anglais) :

http://www.cdc.gov/ncidod/dvrd/spb/mnpages/disinfo.htm

Renseignements sur la soumission d'échantillons à la VSPB :

http://www.cdc.gov/ncidod/dvrd/spb/mnpages/specimen.htm (numéro de téléphone: 404-639-2888)

\section{International}

Site de l'OMS sur les flambées épidémiques:

www.who.int/csr/don/fr

Promed (en anglais) :

www.promedmail.org 


\section{Lectures supplémentaires}

Johnston V, Stockley JM, Dockrell D, et al. Fever in returned travellers presenting in the United Kingdom:

Recommendations for investigation and initial management. J Infection 2009;59:1-18.

Freedman DO, Weld LH, Kozarsky PE, et al. Spectrum of disease and relationship to place of exposure in ill returned travellers. N Engl J Med 2006;354:119-130.

Boggild AK, Page AV, Keystone JS, Morris AM, Liles WC. Delay in diagnosis: malaria in a returning traveller. CMAJ 2009;180(11):1129-1131. Accessible à l'adresse suivante : http://www.cmaj.ca/cgi/content/full/180/11/1129.

Rynor B. Dengue fever on the rise at tourist getaways. CMAJ 2010;182(4):E195-196.

Accessible à l'adresse suivante : http://www.cmaj.ca/cgi/content/full/182/4/E195.

Szakacs TA, McCarthy AE. An all-inclusive vacation. CMAJ 2007;177(1):29-31.

Accessible à l'adresse suivante : http://www.cmaj.ca/cgi/content/full/177/1/29.

Daneman N, Slinger R. Tache noire. CMAJ 2008;178(7):841.

Accessible à l'adresse suivante : http://www.cmaj.ca/cgi/content/full/178/7/841. 


\section{Glossaire}

\begin{tabular}{|c|c|}
\hline $\mathrm{ADN}$ & Acide désoxyribonucléique \\
\hline ANEB & Epstein Barr antigène nucléaire \\
\hline ARN & Acide ribonucléique \\
\hline ASPC & L'Agence de la santé publique du Canada \\
\hline CCMTMV & Le Comité consultatif de la médicine tropical et de la médicine des voyages \\
\hline CDC & Center for Disease Control and Prevention \\
\hline CMV & Cytomégalovirus \\
\hline DV & Diarrhée du voyageur \\
\hline ELISA & Dosage immunoenzymatique \\
\hline GG/CT & Gonorrhée (gonocoque) / Chlamydia trachomatis \\
\hline $\mathrm{HBcAb}$ & Anticorps nucléocapsidique \\
\hline $\mathrm{HBeAb}$ & Anticorps e \\
\hline $\mathrm{HBeAg}$ & Antigène e \\
\hline $\mathrm{HBsAb}$ & Anticorps de surface \\
\hline HBsAg & Antigène de surface \\
\hline $\operatorname{Ig} G$ & Immunoglobuline $G$ \\
\hline $\operatorname{IgM}$ & Immunoglobuline $\mathrm{M}$ \\
\hline ITS & Infection transmissible sexuellement \\
\hline IV & Intraveneuse \\
\hline IVR & Infection des voies respiratoires \\
\hline LCR & Liquide céphalorachidien \\
\hline MII & Maladie intestinale inflammatoire \\
\hline OMS & L'Organisation mondiale de la santé \\
\hline PCR & Amplification en chaîne par polymérase \\
\hline RT & Transcription inverse \\
\hline SARM & Staphylococcus aureus résistant à la méthicilline \\
\hline SASM & Staphylococcus aureus sensible à la méthicilline \\
\hline TB & Tuberculose \\
\hline TVP & Thrombose veineuse profonde \\
\hline VCA du VEB & Virus d'Epstein Barr virus antigène capsulaire \\
\hline VEB & Virus d'Epstein-Barr \\
\hline VHA & Virus de l'hépatite A \\
\hline VHB & Virus de l'hépatite B \\
\hline VHC & Virus de l'hépatite C \\
\hline VHD & Virus de l'hépatite D \\
\hline VHE & Virus de l'hépatite E \\
\hline VHS & Virus de l'herpès simplex \\
\hline VIH & Virus de l'immunodéficience humaine \\
\hline $\mathrm{VNO}$ & Virus du Nil occidental \\
\hline
\end{tabular}




\section{Références}

1. Comité consultatif de la médecine tropicale et de la médecine des voyages. Recommandations canadiennes pour la prévention et le traitement du Paludisme (Malaria) chez les voyageurs internationaux. RMTC 2009;35S1:1-45.

2. Steffen R, Rickenbach M, Wilhem U, Helminger A, Schar M. Health problems after travel to developing countries. J Infect Dis 1987;156:84-91.

3. Hill D. Health problems in a large cohort of Americans traveling to developing countries. J Travel Med 2000;7:259-266.

4. Wilson M, Weld L, Boggild A et al. Fever in returned travelers: results from the GeoSentinel Surveillance Network. Clin Infect Dis 2007;44:1560-1568.

5. Bottieau E, Clerinx J, Schrooten W et al. Etiology and outcome of fever after a stay in the tropics. Arch Intern Med 2006;166:1642-1648.

6. Doherty J, Grant A, Bryceson A. Fever as the presenting complaint of travellers returning from the tropics. QJM 1995;88:277-281.

7. O'Brien D, Tobin S, Brown G, Torresi J. Fever in returned travelers: review of hospital admissions for a 3-year period. Clin Infect Dis 2001;33:603-609.

8. Antinori S, Galimberti L, Gianelli E et al. Prospective observational study of fever in hospitalized returning travelers and migrants from tropical areas, 1997-2001. J Travel Med 2004;11:135-142.

9. Parola P, Soula G, Gazin P, Foucault C, Delmont J, Brouqui P. Fever in travelers returning from tropical areas: prospective observational study of 613 cases hospitalised in Marseilles, France, 1999-2003. Travel Med Infect Dis 2006;4:61-70.
10. West N, Riordan F. Fever in returned travellers: a prospective review of hospital admissions for a 2(1/2) year period. Arch Dis Child 2003;88:432-434.

11. Klein J, Millman G. Prospective, hospital based study of fever in children in the United Kingdom who had recently spent time in the tropics. BMJ 1998;316:1425-1426.

12. Hagmann S, Neugebauer R, Schwartz E et al. Illness in children after international travel: analysis from the GeoSentinel Surveillance Network. Pediatrics 2010;125:e1072-e1080.

13. Freedman DO, Weld LH, Kozarsky PE et al. Spectrum of Disease and Relation to Place of Exposure among Ill Returned Travelers. N Engl J Med 2006;354:119-130.

14. Cooke F, Day M, Wain J, Ward L, Threlfall E. Cases of typhoid fever imported into England, Scotland and Wales (2000-2003). Trans R Soc Trop Med Hyg 2007;101:398-404.

15. Freedeman DO. Malaria prevention in short-term travellers. N Engl J Med 2008;359:603-612.

16. Centers for Disease Control and Prevention. CDC surveillance summaries - malaria surveillance: United States 2004. MMWR 2006;55:23-37.

17. Behrens R, Collins M, Botto B, Heptonstall J. Risk for British travellers of acquiring hepatitis A. BMJ 1995;311:193. 